\title{
THE EFFECT OF SINGLE NUCLEOTIDE POLYMORPHISM (SNP) IN GLIOBLASTOMA MULTIFORME
}

\author{
This is a Pre-Print, Not Peer Reviewed
}

\section{Authors:}

Asmita Ghosh, Department of Biotechnology, Heritage Institute of Technology, Maulana Abul Kalam Azad University of Technology (MAKAUT), WB, asmitaghosh19@gmail.com

*Dattatreya Mukherjee, MBBS Student and Research Assistant, International School Jinan University, P.R China, dattatreyamukherjee4u@outlook.com

Parth Patel, H. K. College of Pharmacy, Jogeshwari West, Mumbai-400102, Maharashtra, India

Parth.patel@hkcp.edu.in

Debraj Mukhopadhyay, ept. of Public Health, School of Allied Health Sciences, Delhi Pharmaceutical Sciences and Research University (DPSRU), Govt. of N.C.T Delhi, New Delhi - 110017.

Debraj364@gmail.com

*Corresponding Author: Dattatreya Mukherjee, MBBS Student and Research Assistant, Jinan University, P.R China, dattatreyamukherjee4u@outlook.com

Copy Right: Asmita Ghosh et al. 


\section{ABSTRACT:}

Single nucleotide polymorphism is a genetic substitution of a base pair at a single position of the genome. SNPs are a common phenomenon and influence mRNA expression. Half of the SNPs occur in the noncoding region with $25 \%$ being mis-sense mutation and $25 \%$ being silent mutations. SNPs belong to the last generation of molecular markers which is identified through SNP mapping. SNPs are extensively studied to distinguish genetic expression and protein synthesis. These genetic differences are a major source of diseases in humans like cancers. One of the most common types of cancer of the brain is the Glioblastoma Multiforme that accounts for more than $80 \%$ of the malignant primary brain tumors (PBT). Researchers have found out a potential role of various SNPs in the genome to have a strong relation with Glioma formation and proliferation. Most SNPs are either not discovered, or their biological mechanisms are unknown, making it difficult to link putative associations with disease onset. The given review aims to identify some of the most common SNPs associated with GBM and classify the genetic basis along with future prospects. These SNPs are pioneer in Genome Wide Association studies to help in cancer research and identification of specific genetic alterations liked to GBM. Single Nucleotide Polymorphisms in a gene can be used as genetic biomarkers to aid better understanding of the mechanism of cancer formation, its aetiology, progression and metastatic behaviour.

Keywords: Single Nucleotide Polymorphisms, Glioblastoma Multiforme, gene mutations, Signalling pathways, Genetic Biomarkers, Genome Wide Association Studies

\section{INTRODUCTION:}

Single Nucleotide polymorphism, also known as SNPs, is the result of a genetic substitution at a definite position on the genome with a mutational rate (mostly transition or transversion) of 1 in 1000 base pairs. SNPs plays a major contribution in gene regulation, where they are capable of altering an encoded amino acid sequence (nonsynonymous) or have high prevalence in the non coding region, thus proving to be a silent gene (synonymous). SNPs are widely studied to distinguish genetic expression, DNA and mRNA conformational changes, protein synthesis, and sub-cellular localization of mRNA and stability of a protein [1]. SNPs have a wide range of usage in the molecular biology and health science field such as in the detection of mutated genes and associated diseases, and as biomarkers. Numerous researches aimed at identification of SNPs have aided the discovery of drugs based on human response, as well as identify specific associated diseases like diabetes, respiratory and heart diseases, rheumatoid arthritis; Alzheimer's and even cancers [2]. SNPs have a significant impact on the formation and proliferation of cancers like Glioblastoma and breast cancer. Glioblastoma Multiforme (GBM) is one of the most aggressive types of tumor associated with the brain and spinal cord with survival duration of 12 to 15 months post-diagnosis with a population of only $3-7 \%$ surviving longer than 5 years [3].

Gliomas are grade IV tumors that constitute $80 \%$ of all malignant primary tumors (PBTs) [4]. Numerous identified SNPs show association with Glioblastomas specifically in high abundance in the promoter region causing polygenetic susceptibility to glioma, as observed from Genome-Wide Association Studies (GWASs) [5]. 
The following review aims to shortlist some of the most common and important SNPs that are actively involved in Glioblastoma development and proliferation.

\section{GENES AND THEIR CORRESPONDING SNPS ASSOCIATED WITH THE RISK OF GLIOBLASTOMAS}

\section{MDR1 gene}

The MDR1 gene is found in exon 12, 21, and 26 in the tumor specimens of patients with glioblastoma [6]. It is multidrug resistance (MDR) 1 gene that encodes a 170-kDa membrane transporter called P-glycoprotein. MDR1 has a vital role in defending cells against lipophilic xenobiotics via an ATP-dependent cellular efflux system [7]. The genome-wide mapping of the MDR1 gene proved the possibility that SNPs in this gene lead to the progress of cancers specifically Glioblastoma. A multivariate investigation of MDR1 genotypes showed that the C/C variant of the exon12 C1236T SNP is prognostic for the continued existence of patients treated with Temozolomide. Temozolomide is a chemotherapeutic drug used for the treatment of GBM and Anaplastic Astrocytomas and is specifically designed to shrink the tumor by killing cancerous cells. Systemic screening of this gene by Hoffermeyer et al. led to the identification of SNPs at position 3435 located in exon 26 in the P-glycoprotein function [7]. The most recurrent SNP MDR1 2677G > T/A in exon 21 , causes the amino acid substitution from Alanine to Serine or Threonine. The third frequent polymorphism of the ABCB1/MDR1 gene is a silent mutation in exon 12 MDR1 1236C > T [7]. Such mutations have led to altered transport activity among genetic variants, thereby reducing the capacity of cells from preventing harmful xenobiotic compounds, and increased cytotoxicity from chemotherapeutic drugs [7, 8]. According to the research of (Oberstadt, 2013), ABCB1 is expressed both in low-grade glioma as well as in high-grade glioma such as glioblastoma. ABCG 2 was established to be expressed in glioma stem cells and in endothelial cells of the large vessels of glioma tissue with potential resistance factors [9]. Few CpGs (5 in MGMT; 2 in ABCB1 and 3 in ABCG2) have been identified in pyrosequencing assays that suggest that methylation in such $\mathrm{CpG}$ sites has a reasonable impact on GBM prognosis. The overall methylation rate in such SNP regions affects the survival rate of patients suffering from GBM [9]. Thus, SNP in MDR1 gene is a novel predictive factor related to patients with Glioblastomas.

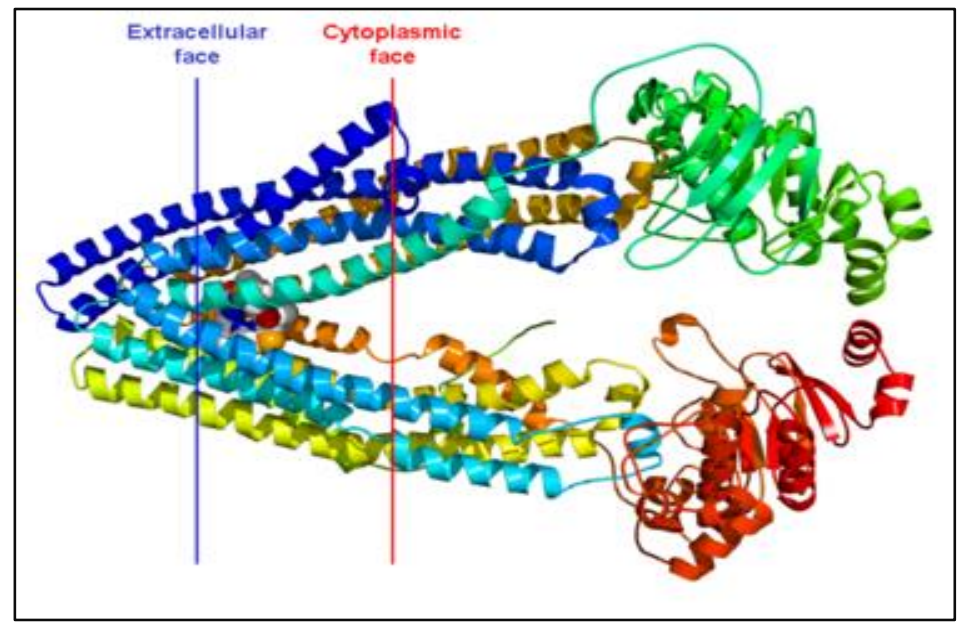

Fig 1:- P-glycoprotein 1 also known as multidrug resistance protein 1 (ABCB1 gene)

[Source: - https://en.wikipedia.org/wiki/P-glycoprotein] 


\section{MDM2 gene:}

Glioblastoma progression from low-grade Astrocytoma is commonly associated with a mutation in the p53 gene (mutation at the high conserved region of exon 5-8) that results in deregulation of cell proliferation and inability to initiate apoptosis. The dysfunction of the TP53 pathway is a common cause of Glioblastoma Multiforme. The MDM2 protein plays an important function in the negative regulation of the T53 pathway by promoting ubiquitination and degradation [10]. A complex form between MDM2 and TP53 is transcriptionally inactive in cells that are rich in GC polymorphism. Studies by (Rina G. Khatri, 2008) showed statistical significance between MDM2 SNP309 genotyping in healthy controls and patients with GBM with respect to 3 genotypes (TT, TG, and GG) [10]. Data from multiple research suggested that MDM2 amplification leads to the increase in protein levels in the presence of MDM2 SNP309 suggesting a high clinical correlation between MDM2 SNP309 and GBM occurrence [10,11]. A higher frequency of G allele in MDM2 SNP309 gene in the pathogenesis of GBM than normal genome complex in healthy subjects was observed [10]. This also proved that the MDM2 SNP309 gene can be used as a biomarker for GBM prognosis for patients with a decreased expression of TP53 due to heightened MDM2. The amplified prevalence of the mutant $G$ allele in patients with GBM and its influence on age of onset imply a possible role in the molecular pathogenesis of GBM, thus may act as a novel therapeutic target [11]. A plausible compound effect between the MDM2 309GG and p53 Pro/Pro genotype showed high clinical significance.

\section{EGRF gene:}

Numerous study reports have shown Single Nucleotide Polymorphism on Epidermal Growth Factors Receptor (EGRF) gene, to play a major role in multiple cancer forms by hindering important cellular responses. Certain genotypes of the EGFR gene may be related to elevated glioblastoma risk, indicating that germline EGFR polymorphisms have a major contribution to carcinogenesis [12]. According to the research of (Hai-Bo Liu 1, 2012), 9 SNPs in 8 genes were studied using the Sequenom MassARRAY platform and Sequenom Assay software [12]. The rs730437 and rs1468727 in ERGF are associated with glioma risk. Studies also revealed that the haplotype "CGTC" increased the risk of developing glioma by $36 \%$ in the EGRF gene [13]. An excess of gene copy number in the EGRF gene was identified in GBM patients by using qPCR analysis [12]. qPCR results also confirmed high EGFR copy number in cases of double minutes, and low values of copy number insertions in gene occur [13]. In most patients, there is a trisomy/polysomy of chromosome 7 , thereby suggesting that this alteration could be a preliminary event in the tumorigenesis of glioblastoma.

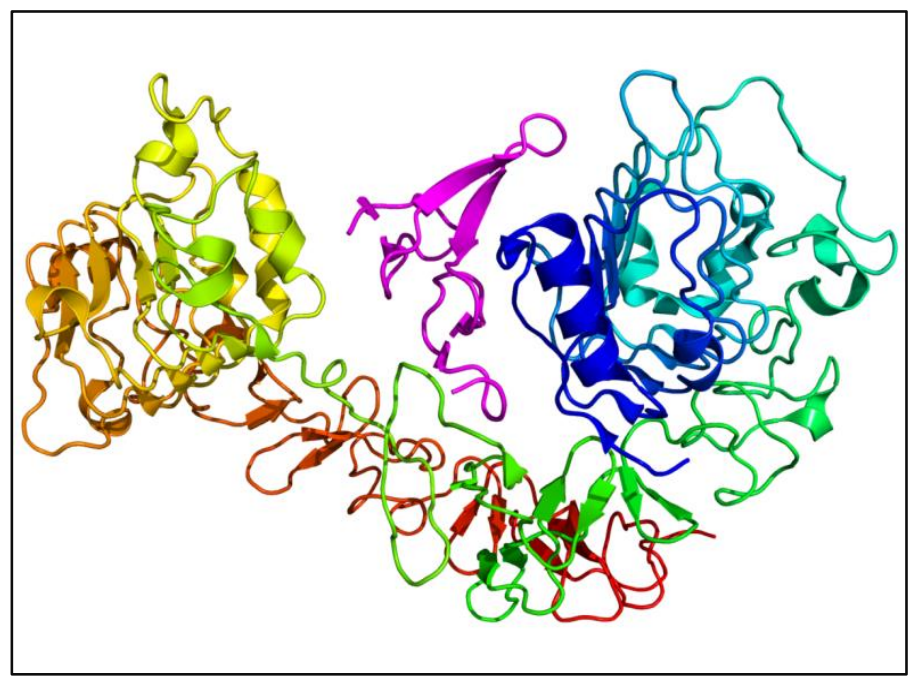

Fig 2: Structure of Epidermal Growth Factor Receptor [Source: - https://en.wikipedia.org/wiki/Epidermal_growth_factor_receptor] 


\section{Kinase-anchored protein 6 (AKAP6) gene}

The A-kinase anchor proteins (AKAPs) are a group of structurally varied proteins which function as a regulatory subunit of protein kinase A (PKA) and bind the holoenzyme to distinct locations within the cell. It is mainly involved in various signal transduction pathways. The study by (Ming Zhang1, 2019) identified five SNPs in the AKAP6 gene through genotyping. The 5 SNP variants were rs1957021, rs2145587, rs2239647, rs4261436 and rs17522122 that might function as enhancer histone markers or by motif conformational change [13]. A relationship between the AA genotype of rs2239647 and increased glioma risk was deduced. SNPs rs2239647, rs4261436, and rs17522122 were extensively associated with the prognosis of patients suffering from GBM and affected gene expression [14]. The regulation of the cAMP/PKA pathway and selective targeting of this signaling pathway through a precise targeted and approach may be a possible target for the treatment of malignant glioma.

\section{Exosomal SOX2 DNA}

Exosomes originated from cancer cells, including GBM, have NANOG and NANOGP8 DNA associated with them. SOX2 is misregulated in cancer cells leading to alterations in miRNA function such as SNPs in the binding sites [15]. SOX2 is an intronless gene that belongs to the SRY-related HMG-box (SOX) family of transcription factors. Polymorphism in this gene has proven to result in lung, breast, and brain cancers [16]. Microarray analysis by (Wen-Shin Song 1, 2016) revealed that mutation in the SOX2 led to Glioblastomas in cancer stem cells, thus providing a novel therapeutic target to overcome the poor survival rate of GBM patients [17]. Micro RNA-induced modulations of SOX2 expression levels have a vital correlation to various types of cancers. Single nucleotide polymorphism in the SOX2 gene has led to the destruction or modification of that gene region leading to cancer vulnerability. SOX2 gene amplification has a high contributing factor toward upregulation of promotor hypo-methylation, metastasis, and invasive migration of glioma [15, 17]. Studies by Fang et al. revealed that SOX2 regulates the expression of 105 precursor miRNAs corresponding to 95 mature miRNAs, whose expression varies with a SOX2 knockdown in GBM. Thus, an expression control loop exists between the SOX2 and miRNAs leading to polygenetic disorders. GBM exosomal DNA shows 1 SNP occurring due to the deletion of flanking nucleotide "C"). SNP binding sites at the miR-145: SOX2 and miR-145 resulted in a doublenegative feedback loop in GBM cell lines. The selective target of miR-145 at the SOX2 gene has led to a decrease in its expression. This results in the misregulation of cancer cells leading to gliomas.

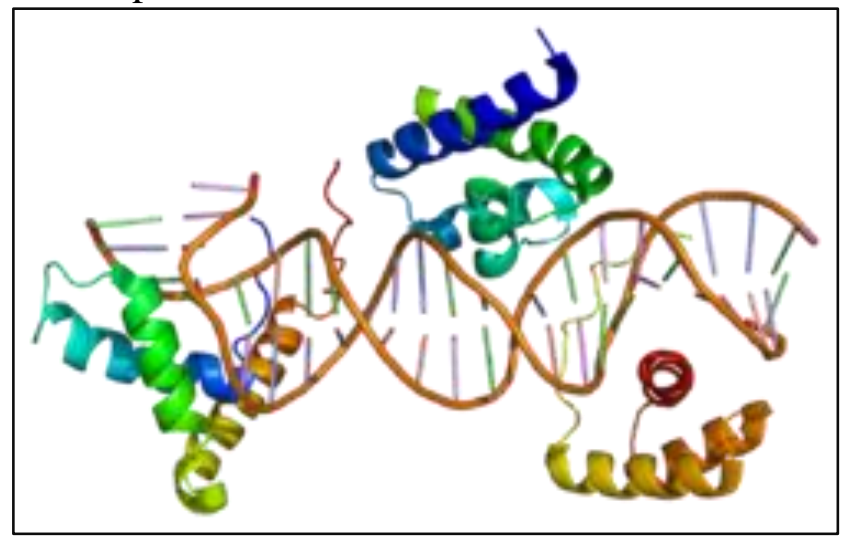

Fig 3:- Molecular Structure of SOX2 [Source: https://en.wikipedia.org/wiki/SOX2]

\section{Cytokine gene:}

Multiple studies supported the relationship between the role of the immune system and GBM growth and proliferation. An inverse association has been proved by (Judith Schwartzbaum, 2005) between allergic conditions and GBM risk, and found classic evidence of relations between immunologic biomarkers and 
glioma risk [19]. Research on allergic conditions and polymorphism variations leading to glioma development led to the finding of 2 SNPs related to brain tumor formation. The Ser478Pro and Gln551Arg on the interleukin (IL-4) receptor $\alpha$ gene (IL-4RA) are associated with increased allergic conditions in case of GBM. The T1 polymorphism found in the asthma gene, ADAM33 is a metalloprotease family member, and has contribution in the matrix degradation and GBM invasion [20]. Asthma susceptibility polymorphism has an inverse relation to asthma and GBM [19]. In 58\% of asthma cases, 80\% of GBM cases have the CC genotype for the IL-13-1,112 C/T polymorphism [19]. A total of three biomarker associations have been reported from the studies of (Judith Schwartzbaum, 2005) which includes IL-4RA Ser478Pro TC, CC; IL4RA Gln551Arg AG, AA; and IL-13 -1,112 CT, TT. These results can be used as a prognostic tool for biomarker in GBM $[19,20]$. According to the studies of A.V.Brenner et al., who genotyped 11 SNPs in 7 cytokine genes, IL4 (rs2243248, 21098T>G) and IL6 (rs1800795, 2174G>C) polymorphisms were extensively connected with the possibility of glioma in patients [21]. Genetic association studies found an important link between polymorphisms in cytokine genes and GBM. Thus, these SNPs can act as an essential biomarker for clinical studies of patients with Glioblastomas.

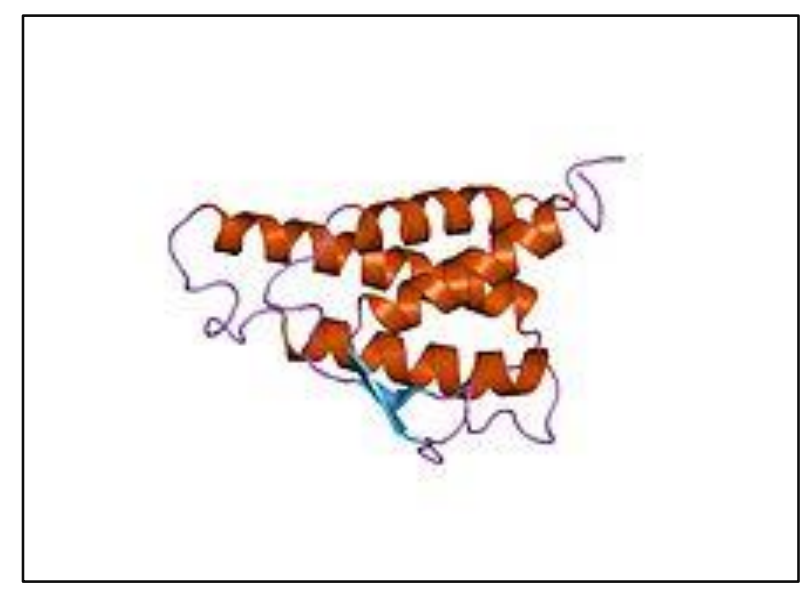

Fig 4: Human Interleukin 4 crystal structure [Source: https://en.wikipedia.org/wiki/Interleukin 4]

\section{PARP1 gene}

The PARP1 gene encodes the Poly [ADP-ribose] polymerase 1 (PARP-1) protein, and is also known as $\mathrm{NAD}^{+}$ADP-ribosyltransferase 1 or poly [ADP-ribose] synthase 1 [22]. PARP1 gene has varied functions like DNA damage repair, proliferation, and tumor transformation, as well as stimulation of inflammation. PARP1 has a crucial role in the base excision repair pathway with functional variations in various cancer subtypes. The research of (Khan, 2019) identified three SNPs in the PARP1 gene namely rs1136410 (Val762Ala), rs1805404 (Asp81Asp), and rs1805414 (Ala284Ala) using AS-PCR and DNA sequencing techniques in patients suffering from Glioblastomas. The rs1805414 (Ala284Ala) in exon 7 is present at position 284 within the PADR-1 domain [22]. The SNP rs1805404 (Asp81Asp) is present in exon 2 at position 81 within the zinc finger domain. The SNP rs1136410 leads to substitution of valine by alanine at codon 762 of the catalytic domain which results in decreased activity of Poly ADP ribosylation, hence playing a major factor in the formation of brain tumors [23]. The reports from the study of (Liu, 2016; Liu, 2016), states that gene carriers who carry forward polymorphism rs1136410 in the T allele at the PARP1 gene, are more prone to develop Gliomas, especially in the Caucasian

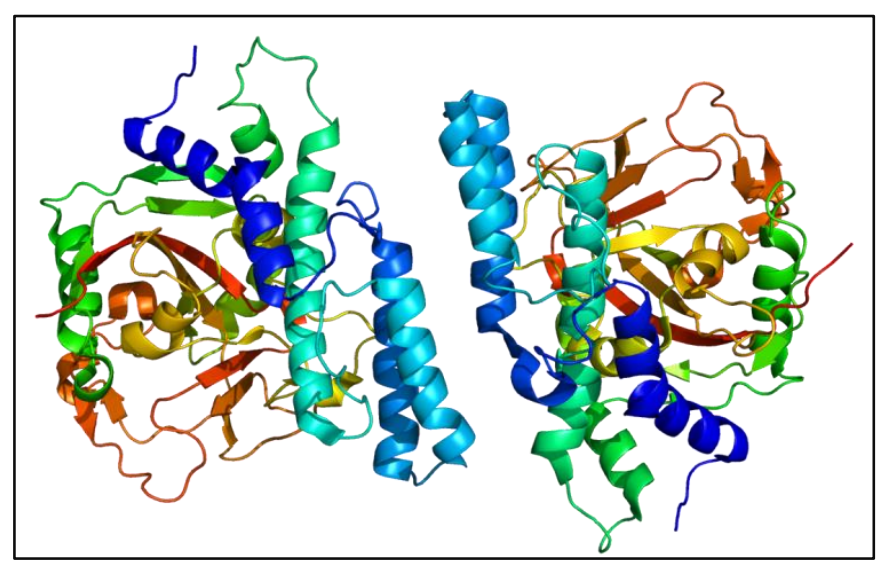

Fig5: Molecular structure of PARP1 gene [Source: https://en.wikipedia.org/wiki/PARP1] 
race. It leads to a substitution of Cytosine to Thymine base, thus reducing the activity of poly (ADPribosyl)ation and also decrease protein expression [24]. Further research to ascertain these SNPs' role as a prognostic biomarker for GBM detection is warranted.

\section{XRCC gene}

The XRCC1 gene, also known as X-ray repair cross-complementing protein 1, is a DNA repair protein, involved in DNA repair in association with DNA ligase III [25]. It participates in base excision repair, and nucleotide excision repair through enzymatic action that repairs single- stranded breaks in the DNA [25, 26]. Single Nucleotide Polymorphism in this gene results in overexpression of this gene ultimately leading to cancers. SNP genotyping of XRCC1 $\operatorname{Arg} 194 \operatorname{Trp}(\mathrm{C} / \mathrm{T})$ at exon 6 (rs1799782), Pro206Pro (A/G) at exon 7 (rs915927), Arg280His (G/A) at exon 9, and Arg399Gln (G/A) at exon 10 (rs25487) are known to be important biomarkers for GBM analysis [26]. In the case of XRCC3, Thr241Met and rs861539 SNP pose a threat to the normal functioning of the XRCC3 gene, thereby leading to increased risk of Glioblastomas especially in the Caucasian population [27. 28]. The rs 1805377 of XRCC4 polymorphism has shown to cause brain tumors in Asian populations too [24]. Very few studies established the relationship between glioma association and XRCC1 and XRCC3 SNPs, hence detailed research work is necessary to establish definite relationships regarding other possible polymorphisms. Two homozygous variant carrier genotypes (XRCC1 Gln399Gln and XRCC3 Met241Met) seem to have a largely increased risk of glioma and meningioma according to the studies of (Kiuru, 2008) [28]. However, extended studies are considered necessary to evaluate gene-environment interactions in DNA repair gene polymorphisms and the possibility of glioma in order to explore the mechanisms through which these polymorphisms influence cancer propensity.

\section{AKT gene}

The AKT gene is RAC gamma-Serine/Threonine protein kinases. It is pivotal in the regulation of cell signaling in response to insulin and growth factors and aid in tumorigenesis, apoptosis, and glycogen synthesis and cell differentiation [29]. Akt is known to be robust oncogenes that have a major contribution to glioma formation. Single nucleotide polymorphisms in the Akt gene assist in tumor recurrence and resistance to DNA damage in mutated tumor cells [30]. Akt family consists of three gene members namely Akt1, Akt2, and Akt3. According to (Hideo Mure, 2009 )'s research, Akt2 and Akt3 play an important role in the viability of human malignant glioma cells [31]. AKT3 is greatly expressed in glioma and plays a central role in tumor viability by activating DNA repair [32]. One of the foremost downstream effectors of phosphatidylinositol 3-kinase which is highly expressed during active neurogenesis, is the polymorphism at $1 \mathrm{q} 44$ rs 12076373 that maps to the eighth intron of AKT3 (v-akt murine thymoma viral oncogenes homolog 3) [33]. SNPs in the AKT3 gene lead to overexpression of this gene and thus Glioma proliferation. In the treatment of Glioblastomas with Temozolomide or radiation, Atk3 activates the DNA repair pathway that enhances the survival of glioma cells [30]. Hence, blocking this repair pathway will facilitate the prevention of DNA repair-mediated therapeutic resistance. 


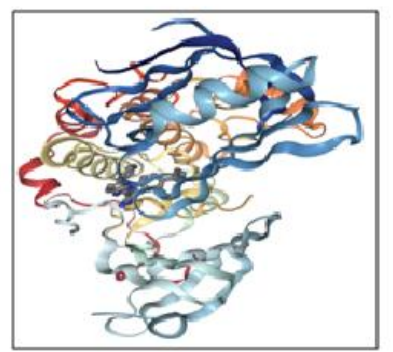

Fig 6:- AKT1 gene structure

[Source:

https:/www.sinobiological.com/resource/akt1/ proteins\#pid=1]

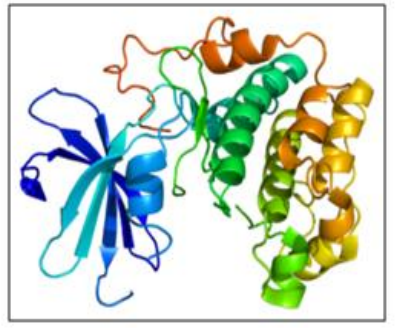

Fig 7: AKT2 gene structure [Source: https://en.wikipedia.org/wiki/AKT2]

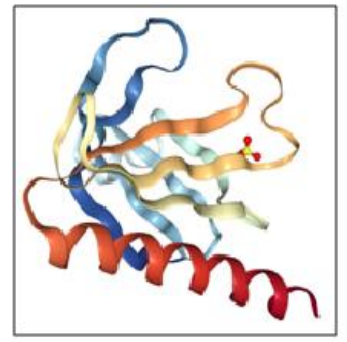

Fig 8: AKT3 gene struture

Source:https://www.sinobiological.com/resource/a kt $3 /$ proteins

\section{IDH gene}

IDH gene codes for the protein Isocitrate dehydrogenase. Mutation (epigenetic alterations) in the IDH leads to brain tumor formations. More than $90 \%$ of the IDH mutations occur at codon $\mathrm{R}_{132}$ of the IDH1 gene. It leads to the production of 2-hydroxyglutarate which is an oncometabolite. This in turn induces DNA methylation called G-CIMP and histone methylation which promotes the formation of Glioblastomas [34]. Studies reveal IDH mutation in Grade II and III gliomas in more than $80 \%$ of the patients suffering from secondary GBM [35]. All studied mutations have been a single amino acid missense mutation in IDH1 at arginine 132 (R132) or in IDH2 (R172) in the form of Single Nucleotide Polymorphisms. (Parsons, 2008), identified major pathways involved in nervous system signaling like TP53, RB1, and PI3K that are affected due to this alteration in the gene [36]. Recurrent mutations were also discovered in $12 \%$ of the GBM patient at their IDH1 genes [36]. SNP at codon 132 in IDH1 exhibits mutations in 395G>A (R132H) substitution and at R132C. In the case of IDH2, mutations were identified at codon 172 in gliomas but with a lower mutational frequency than IDH1especially in the younger generations [34, 35]. Thus, further research will help in genomic analysis to identify potential useful genomic alterations that can help in targeted therapy of GBM. Thus, SNP identification has clinical prognostic importance and aid the identification of genetic and epigenetic alterations in molecular subtypes of GBM.

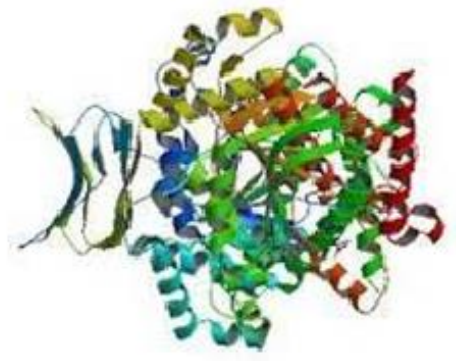

Fig 9: IDH1 gene structure Source: https:/www.phosphosite.org/proteinAction? id=10630\&showAllsites=true

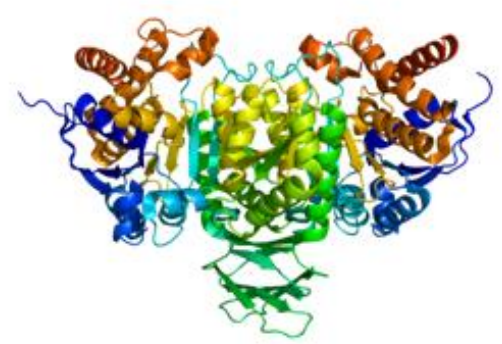

Fig 10: : IDH2 gene structure Source: $h$ ttps:/commons. wikimedia.org/wiki/File:Protein $\mathrm{DH} 2$ PDB 1lwd.png 


\section{RESULT AND DISCUSSION}

A total of 10 genes at their SNPs at different loci have been discussed. These SNPs have proven to be an integral reason for tumor formation and metastasis, as well as lead to the development of Glioblastoma Multiforme. Since the advent of Genome Wide Associaation Studies (GWAS), germline variants have been identified that have had association with Glioblastoma susceptibility [37]. In spite of such explicit and detailed research, a lot is yet left to unravel. Future efforts warrants more focus on assessing association of germline possibility of SNPs with particular molecular subgroups of glioma, as well as investigate the practical basis of the risk loci in tumor formation. Other sequencing tools like high throughput sequencing have opened avenues for genetic screening and SNP identification that indicates the probability of genetic diseases like GBM [38]. Current studies have proofed to be a strong reference for future research that needs to put more focus on tumor formation and aetiology, its associated signaling pathways as well as their clinical translations. In association with the studies related to Glioma formation, sequence based researches have also found association of the above mentioned SNPs with other cancerous formations in the body with majority of the genetic components explained by polygenetic contributions by Single Nucleotide Polymorphisms [37]. The understanding of molecular basis and its various reactions to environmental stimuli will provide scientists better understanding of its mechanism and transformations. Therefore, it will help in finding potential and novel therapeutic applications that can pave way for new dimensions of cure and drug designing. The field of epigenetic regulation of diverse genome components can be enhanced if accurate and precise sequencing is achieved [39]. Many issues require further research, such as the ascertainment prejudice of well known biparental populations and the minimum validation pace of some array-based genotyping platforms [39].

\section{CONCLUSION}

Our study is aimed to analyze the association between various SNP genotypes and its impact on considerable variability of anatomical volumes of cancerous growth in patients suffering from Glioblastoma Multiforme. Our finding underlines the importance of various SNPs and how they might be useful in biomarker studies as novel therapeutic targets. Various mechanisms of SNP function are yet to be uncovered [39, 40]. Our primary source of data is the Cancer Genome Atlas (TCGA) which has generated a complete catalog of molecular alterations and tamper signaling pathways in GBM patients [40]. The GWAS has also helped in our preliminary studies to assess molecular structure and histopathological data related to oncogenes and SNPs in Gliomas. The Next Generation Sequencing studies have shown that the most clinically relevant genes for brain tumor initiation and metastasis are T53, IDH1, IDH2, EGRF and MDM2 [40, 41]. NGS tools can refine intrinsic diagnosis of primary brain tumors by detecting various genetic abnormalities.

Breakthrough research in genomics studies through sequencing and computational software will help in selecting the most appropriate therapeutic approach and also prove to be beneficial in molecular modeling and drug designing techniques. Additionally, latest genetic lesions recognized by SNP chip could become targets for novel therapies [42]. The SNP- Chip provides with consolidated results in terms of genetic abnormalities including duplications, deletions, transversions, polysomy, and Loss of Heterozygosity (LOH) with normal copy number. 


\section{References:}

1. Shastry, B. S. (2009). SNPs: Impact on Gene Function and Phenotype. Single Nucleotide Polymorphisms, 3-22. doi:10.1007/978-1-60327-411-1_1

2. https://medlineplus.gov/genetics/understanding/genomicresearch/snp/

3. https://en.wikipedia.org/wiki/Glioblastoma

4. Ben Kinnersley1, Richard S Houlston1 and Melissa L Bondy2; Genome-wide association studies in glioma; 2018 American Association for Cancer Research ; DOI: 10.1158/1055-9965.EPI-17-1080

5. Kinnersley, B., Labussière, M., Holroyd, A., Di Stefano, A.-L., Broderick, P., Vijayakrishnan, J.,Houlston, R. S. (2015). Genome-wide association study identifies multiple susceptibility loci for glioma. Nature Communications, 6(1). doi:10.1038/ncomms9559

6. Schaich, M., Kestel, L., Pfirrmann, M., Robel, K., Illmer, T., Kramer, M., ... Krex, D. (2008). A MDR1 (ABCB1) gene single nucleotide polymorphism predicts outcome of temozolomide treatment in glioblastoma patients. Annals of Oncology, 20(1), 175-181. doi:10.1093/annonc/mdn548

7. Wang, LH., Song, YB., Zheng, WL. et al. The association between polymorphisms in the MDR1 gene and risk of cancer: a systematic review and pooled analysis of 52 case-control studies. Cancer Cell Int 13, 46 (2013). https://doi.org/10.1186/1475-2867-13-46

8. Gréen, H., Falk, I. J., Lotfi, K., Paul, E., Hermansson, M., Rosenquist, R., ... Nahi, H. (2010). Association of $\mathrm{ABCB} 1$ polymorphisms with survival and in vitro cytotoxicty in de novo acute myeloid leukemia with normal karyotype. The Pharmacogenomics Journal, 12(2), 111-118. doi:10.1038/tpj.2010.79

9. Oberstadt, M. C., Bien-Möller, S., Weitmann, K., Herzog, S., Hentschel, K., Rimmbach, C., Kroemer, H. K. (2013). Epigenetic modulation of the drug resistance genes MGMT, ABCB1 and ABCG2 in glioblastoma multiforme. BMC Cancer, 13(1). doi:10.1186/1471-2407-13-617

10. Rina G. Khatri, B.S., Kapila Navaratne, M.S., and Robert J. Weil, M.D. The role of a single nucleotide polymorphism of MDM2 in glioblastoma multiforme; J Neurosurg 109:000-000, 2008

11. Wan, Y., Wu, W., Yin, Z., Guan, P., \& Zhou, B. (2011). MDM2 SNP309, gene-gene interaction, and tumor susceptibility: an updated meta-analysis. BMC Cancer, 11(1). doi:10.1186/1471-2407-11-208

12. Lopez-Gines, C., Gil-Benso, R., Ferrer-Luna, R., Benito, R., Serna, E., Gonzalez-Darder, J., CerdáNicolas, M. (2010). New pattern of EGFR amplification in glioblastoma and the relationship of gene copy number with gene expression profile. Modern Pathology, 23(6), 856-865. doi:10.1038/modpathol.2010.62

13. Hou, W.-G., Ai, W.-B., Bai, X.-G., Dong, H.-L., Li, Z., Zhang, Y.-Q., \& Xiong, L.-Z. (2012). Genetic Variation in the EGFR Gene and the Risk of Glioma in a Chinese Han Population. PLoS ONE, 7(5), e37531. doi:10.1371/journal.pone.0037531

13. Hai-Bo Liu ${ }^{1}$, Yu-Ping Peng, Chang-Wu Dou, Xiu-Lan Su, Nai-Kang Gao, Fu-Ming Tian, Jie Bai; Comprehensive study on associations between nine SNPs and glioma risk; Asian Pac J Prev Cancer 2012;13(10):4905-8. ; doi: 10.7314/apjcp.2012.13.10.4905. 
14. Zhang, M., Zhao, Y., Zhao, J., Huang, T., \& Wu, Y. (2019). Impact of AKAP6 polymorphisms on Glioma susceptibility and prognosis. BMC Neurology, 19(1). doi:10.1186/s12883-019-1504-2

15. Vaidya, M., \& Sugaya, K. (2020). Differential sequences and single nucleotide polymorphism of exosomal SOX2 DNA in cancer. PLOS ONE, 15(2), e0229309. doi:10.1371/journal.pone.0229309

16. http://www.cancerindex.org/geneweb/SOX2.htm

17. Song, W.-S., Yang, Y.-P., Huang, C.-S., Lu, K.-H., Liu, W.-H., Wu, W.-W., Chen, M.-T. (2016). Sox2, a stemness gene, regulates tumor-initiating and drug-resistant properties in CD133-positive glioblastoma stem cells. Journal of the Chinese Medical Association, 79(10), 538-545. doi:10.1016/j.jcma.2016.03.010

18. Vaidya, M., \& Sugaya, K. (2020). Differential sequences and single nucleotide polymorphism of exosomal SOX2 DNA in cancer. PLOS ONE, 15(2), e0229309. doi:10.1371/journal.pone.0229309

19. Schwartzbaum, J., Ahlbom, A., Malmer, B., Lönn, S., Brookes, A. J., Doss, H., ... Feychting, M. (2005). Polymorphisms Associated with Asthma Are Inversely Related to Glioblastoma Multiforme. Cancer Research, 65(14), 6459-6465. doi:10.1158/0008-5472.can-04-3728 (Judith Schwartzbaum, 2005)

20. Mitsutoshi Nakada 1 , Yasunori Okada 2 , Junkoh Yamashita 1 THE ROLE OF MATRIX METALLOPROTEINASES IN GLIOMA INVASION; [Frontiers in Bioscience 8, e261-269, January 1, 2003; doi: 10.2741/1016

21. A.V.Brenner_, M.A.Butler1, S.S.Wang, A.M.Ruder2, N.Rothman, P.A.Schulte3, S.J.Chanock4, H.A.Fine5 M.S.Linet and P.D.Inskip Single-nucleotide polymorphisms in selected cytokine genes and risk of adult glioma; Carcinogenesis vol.28 no.12 pp.2543-2547, 2007 doi:10.1093/carcin/bgm210

\section{2. https://www.genecards.org/cgi-bin/carddisp.pl?gene=PARP1}

23. Khan, A. ullah, Mahjabeen, I., Malik, M. A., Hussain, M. Z., Khan, S., \& Kayani, M. A. (2019). Modulation of brain tumor risk by genetic SNPs in PARP1gene: Hospital based case control study. PLOS ONE, 14(10), e0223882. doi:10.1371/journal.pone.0223882

24. Liu, K., \& Jiang, Y. (2016). Polymorphisms in DNA Repair Gene and Susceptibility to Glioma: A Systematic Review and Meta-Analysis Based on 33 Studies with 15 SNPs in 9 Genes. Cellular and Molecular Neurobiology, 37(2), 263-274. doi:10.1007/s10571-016-0367-y

24. Liu, K., \& Jiang, Y. (2016). Polymorphisms in DNA Repair Gene and Susceptibility to Glioma: A Systematic Review and Meta-Analysis Based on 33 Studies with 15 SNPs in 9 Genes. Cellular and Molecular Neurobiology, 37(2), 263-274. doi:10.1007/s10571-016-0367-y

\section{5. https://en.wikipedia.org/wiki/XRCC1}

26. Takeshita, H., Fujihara, J., Yasuda, T., \& Kimura-Kataoka, K. (2014). Worldwide Distribution of Four SNPs in X-Ray and Repair and Cross-Complementing Group 1 (XRCC1). Clinical and Translational Science, 8(4), 347-350. doi:10.1111/cts.12237 
27. Adel Fahmideh, M., Schwartzbaum, J., Frumento, P., \& Feychting, M. (2014). Association between DNA repair gene polymorphisms and risk of glioma: A systematic review and meta-analysis. NeuroOncology, 16(6), 807-814. doi:10.1093/neuonc/nou003

28. Kiuru, A., Lindholm, C., Heinävaara, S., Ilus, T., Jokinen, P., Haapasalo, H., ... Auvinen, A. (2008). XRCC1 and XRCC3 variants and risk of glioma and meningioma. Journal of Neuro-Oncology, 88(2), 135142. doi:10.1007/s11060-008-9556-y

\section{9. https://www.ncbi.nlm.nih.gov/pmc/articles/PMC4371922/}

30. Turner, K. M., Sun, Y., Ji, P., Granberg, K. J., Bernard, B., Hu, L. Zhang, W. (2015). Genomically amplified Akt3 activates DNA repair pathway and promotes glioma progression. Proceedings of the National Academy of Sciences, 112(11), 3421-3426. doi:10.1073/pnas.1414573112

31. Mure, H., Matsuzaki, K., Kitazato, K. T., Mizobuchi, Y., Kuwayama, K., Kageji, T., \& Nagahiro, S. (2009). Akt2 and Akt3 play a pivotal role in malignant gliomas. Neuro-Oncology, 12(3), 221232. doi:10.1093/neuonc/nop026

32. Yan H, Parsons DW, Jin G, McLendon R, Rasheed BA, Yuan W, et al. IDH1 and IDH2 mutations in gliomas. The New England journal of medicine 2009;360(8):765-73 doi 10.1056/NEJMoa0808710.

33. Boland E, Clayton-Smith J, Woo VG, McKee S, Manson FD, Medne L, et al. Mapping of deletion and translocation breakpoints in 1q44 implicates the serine/threonine kinase AKT3 in postnatal microcephaly and agenesis of the corpus callosum. American journal of human genetics 2007;81(2):292-303 doi 10.1086/519999.

34. Tateishi, K., \& Yamamoto, T. (2020). IDH-Mutant Gliomas. Brain and Spinal Tumors - Primary and Secondary. doi:10.5772/intechopen.84543

35. Cohen, A. L., Holmen, S. L., \& Colman, H. (2013). IDH1 and IDH2 Mutations in Gliomas. Current Neurology and Neuroscience Reports, 13(5). doi:10.1007/s11910-013-0345-4

36. Parsons, D. W., Jones, S., Zhang, X., Lin, J. C.-H., Leary, R. J., Angenendt, P., ... Kinzler, K. W. (2008). An Integrated Genomic Analysis of Human Glioblastoma Multiforme. Science, 321(5897), 18071812. doi:10.1126/science. 1164382

37. Ben Kinnersley1, Richard S Houlston1 and Melissa L Bondy2. Genome-wide association studies in glioma; 2018 American Association for Cancer Research; DOI: 10.1158/1055-9965.EPI-17-1080

38. Keller, A., Harz, C., Matzas, M., Meder, B., Katus, H. A., Ludwig, N., ... Meese, E. (2011). Identification of Novel SNPs in Glioblastoma Using Targeted Resequencing. PLoS ONE, 6(6), e18158. doi:10.1371/journal.pone.0018158

39. Kumar, S., Banks, T. W., \& Cloutier, S. (2012). SNP Discovery through Next-Generation Sequencing and Its Applications. International Journal of Plant Genomics, 2012, 1-15. doi:10.1155/2012/831460

40. Sadegh Shirian ${ }^{1,2}$, Yahya Daneshbod ${ }^{3}$, Saranaz Jangjoo ${ }^{4}$, Amir Ghaemi ${ }^{5}$, Arash Goodarzi ${ }^{6}$, Maryam Ghavideldarestani ${ }^{7}$, Ahmad Emadi ${ }^{1}$, Arman Ai ${ }^{8}$, Akbar Ahmadi ${ }^{9}$, , Jafar $\mathrm{Ai}^{9}{ }^{\text {,**}}$ The Role of Next Generation Sequencing in Diagnosis of Brain Tumors: A Review Study; Neuroscience: 7 (1); e68874; doi: 10.5812/ans.68874 
41. Chang, J. S., Yeh, R.-F., Wiencke, J. K., Wiemels, J. L., Smirnov, I., Pico, A. R., ... Wrensch, M. R. (2008). Pathway Analysis of Single-Nucleotide Polymorphisms Potentially Associated with Glioblastoma Multiforme Susceptibility Using Random Forests. Cancer Epidemiology Biomarkers \& Prevention, 17(6), 1368-1373. doi:10.1158/1055-9965.epi-07-2830

42. Yin, D., Ogawa, S., Kawamata, N., Tunici, P., Finocchiaro, G., Eoli, M., ... Koeffler, H. P. (2009). High-Resolution Genomic Copy Number Profiling of Glioblastoma Multiforme by Single Nucleotide Polymorphism DNA Microarray. Molecular Cancer Research, 7(5), 665-677. doi:10.1158/15417786.mcr-08-0270

43. Fig 1: https://en.wikipedia.org/wiki/P-glycoprotein

44. Fig 2: https://en.wikipedia.org/wiki/Epidermal_growth_factor_receptor

45. Fig 3: https://en.wikipedia.org/wiki/Epidermal growth factor receptor

46. Fig 4: https://en.wikipedia.org/wiki/Interleukin_4

47. Fig 5: https://en.wikipedia.org/wiki/PARP1

48. Fig 6: https://www.sinobiological.com/resource/akt1/proteins

49. Fig 7: https://en.wikipedia.org/wiki/AKT2

50. Fig 8: https://www.sinobiological.com/resource/akt3/proteins

51. Fig 9: https://www.phosphosite.org/proteinAction?id=10630\&showAllSites=true

52. Fig 10: https://commons.wikimedia.org/wiki/File:Protein_IDH2_PDB_1lwd.png 